\title{
Retraction of: Association Between Polymorphisms in the Interleukin-10 Gene and Susceptibility to HIV-1 Infection," by Dan-Hui Fu, Wen-Juan Deng, Zhi Yang, Sen Hong, Qian-Ling Ding, Yang Zhao, Jia Chen, and Dan-Ke Su; AIDS Res Hum Retroviruses, epub: 16 Jun 2020; DOI: 10.1089/AID.2020.0011
}

AIDS Research and Human Retroviruses officially retracts the paper entitled, "Association Between Polymorphisms in the Interleukin-10 Gene and Susceptibility to HIV-1 Infection," by Dan-Hui Fu, Wen-Juan Deng, Zhi Yang, Sen Hong, Qian-Ling Ding, Yang Zhao, Jia Chen, and Dan-Ke Su (AIDS Res Hum Retroviruses, epub: 16 Jun 2020; DOI: 10.1089/AID.2020.0011) due to a final, post-acceptance plagiarism review of the paper revealed a level of duplication of published sources that exceeded normal thresholds. The authors were provided an opportunity to adjust the problem, but the revision was returned with an even higher degree of duplication.

The Editor and Publisher of AIDS Research and Human Retroviruses are committed to preserving the scientific literature and the community it serves. 